\title{
Accounting for evolving pore size distribution in water retention models for compacted clays
}

\author{
Gabriele Della Vecchia ${ }^{1, *, \dagger}$, Anne-Catherine Dieudonné ${ }^{2,3}$, Cristina Jommi $^{4}$ \\ and Robert Charlier ${ }^{2}$ \\ ${ }^{1}$ Politecnico di Milano, Milano, Italy \\ ${ }^{2}$ University of Liege, Liege, Belgium \\ ${ }^{3}$ F.R.I.A., Fonds de la Recherche Scientifique - FNRS, Brussels, Belgium \\ ${ }^{4}$ Delft University of Technology, Delft, The Netherlands
}

\begin{abstract}
SUMMARY
Water retention in compacted clays is dominated by multi-modal pore size distribution which evolves during hydro-mechanical paths depending on water content and stress history. A description of the evolutionary fabric has been recently introduced in models for water retention, but mostly on a heuristic base. Here, a possible systematic approach to account for evolving pore size distribution is presented, and its implications in models for water retention are discussed. The approach relies on quantitative information derived from mercury intrusion porosimetry data. The information is exploited to introduce physically based evolution laws for the parameters of water retention models. These laws allow tracking simultaneously the evolution of the aggregated fabric and the consequent hydraulic state of compacted clays. The influence of clay microstructure, mechanical constraints and water content changes on the water retention properties is highlighted and quantified from experimental data on different compacted soils with different activity of the clayey fraction. The framework is discussed with reference to a widespread water retention model and validated against experimental data on a Sicilian scaly clay compacted to different dry densities and subjected to a number of hydro-mechanical paths. Copyright () 2014 John Wiley \& Sons, Ltd.
\end{abstract}

Received 25 March 2014; Revised 28 July 2014; Accepted 20 August 2014

KEY WORDS: compacted clays; water retention model; microstructure; mercury intrusion porosimetry; pore size distribution

\section{INTRODUCTION}

Classical water retention models have been formulated as a unique relationship between soil suction and degree of saturation, $\mathrm{Sr}$, or volumetric water content, $\theta$. Despite their apparent phenomenological nature, the earliest and most diffused analytical proposals have been developed incorporating parameters linked to the pore size distribution of the porous medium, for example Brooks and Corey [1], van Genuchten [2] and Fredlund and Xing [3].

More recently, because of evidence coming from new experimental data, the role of mechanical variables, such as density and stress state, has been recognised, and attention has been paid to their influence on the water retention properties, focussing essentially on the dependence of the air-entry value on the void ratio [4-15]. The nature of the previous relationships is basically phenomenological, in other words related to the hydraulic response of the porous medium at the laboratory scale. However, they implicitly describe the role of microstructure on the hydraulic properties.

*Correspondence to: Gabriele Della Vecchia, Department of Civil and Environmental Engineering, Politecnico di Milano, Piazza Leonardo da Vinci, 32, 20133, Milano, Italy.

†E-mail: gabriele.dellavecchia@polimi.it 
Focussing the attention on compacted clays, it is well recognised that compaction on the dry side of the optimum water content develops an aggregated fabric, characterised at least by two levels of pores: intra-aggregate and inter-aggregate. Experimental evidence shows that this fabric is not unique and that the size of the aggregates is sensitive to the compaction water content [16], to the chemical composition of the pore fluid [17] and to the actual hydromechanical path followed [18]. Furthermore, the fabric developed during compaction is not fixed, but evolves along multiphysics generalised stress paths, as discussed by Romero et al. [19], Cuisinier and Laloui [20], Koliji et al. [21], Della Vecchia [22], Monroy et al. [23] and Wang et al. [24], amongst others. As a consequence, deep comprehension of the soil structure and of its changes along hydro-mechanical paths should be the starting point for the modelling of the retention behaviour of compacted clays, irrespective of the analytical form chosen for the constitutive laws.

The experimental technique most often used to gather quantitative information on the fabric of porous media with interconnected porosity is the mercury intrusion porosimetry (MIP) (see, e.g. the works of Delage and Lefebvre [25], Griffiths and Joshi [26], Al Mukhtar et al. [27], Cuisinier and Laloui [20], Simms and Yanful [28] and Thom et al. [16], amongst others). Detailed information provided by MIP has been exploited by some authors to develop water retention models able to account for evolving fabric. Interesting contributions are provided by Simms and Yanful [29], Romero et al. [30], Casini et al. [31], Beckett and Augarde [32], Della Vecchia et al. [33], Hu et al. [34] and Dieudonné et al. [35]. It is worth remarking, at this stage, that whilst the pore network topology depends on the complex fabric of the different structure levels of a soil, MIP provides only a partial information on the soil fabric, which is a scalar measure of the interconnected pore sizes. Accounting for this limitation, the terms microstructure and macrostructure will be adopted in the following to indicate the distribution of the intra-aggregate (micro)pores and of the inter-aggregate (macro)pores, respectively.

The role of evolving pore size distribution for deformable soils has been discussed by $\mathrm{Hu}$ et al. [34], in modelling the hysteretic water retention behaviour. The latter authors assumed that the shape and the distribution pattern of the pore size density (PSD) function are not significantly altered during deformation processes, and they neglected the change in intra-aggregate pore size distribution. However, along hydro-chemo-mechanical loading paths, changes in the intraaggregate porosity are not negligible when variations of water content and/or chemical composition of the pore fluid are involved.

A methodology is presented herein to take into account the evolution of the pore network, as detected from the pore size distribution, into water retention models in a comprehensive way. An insight into the link between the pore size distribution of a soil and its water retention properties is presented. The framework is firstly introduced for single-porosity media, and it is then extended to double-porosity media such as compacted clays. The influence of mechanical loads and changes in water content on the clay structure, as well as the consequences of this structure on water retention, is emphasised and quantified, based on a detailed analysis of experimental data on different compacted soils with different activity of the clayey fraction. The implications of the proposed framework are highlighted by analysing the theoretical response of the model along hydro-mechanical stress paths. The framework is then validated against experimental data on a compacted Sicilian scaly clay.

\section{PORE SIZE DENSITY FUNCTION AND WATER RETENTION PROPERTIES}

Let $f(r)$ indicate the generic pore size distribution of a porous medium. The relative frequency of pores whose size ranges between $r$ and $(r+d r)$ is equal to $f(r) \cdot d r$, and, by definition,

$$
\int_{0}^{\infty} f(r) d r=1
$$


Indeed, the pore sizes have a lower, $R_{\min }$, and an upper, $R_{\max }$, bound, which allows writing

$$
\int_{R \min }^{R \max } f(r) d r=1
$$

The cumulative function $F(R)$

$$
F(R)=\int_{0}^{R} f(r) d r=\int_{R \min }^{R} f(r) d r
$$

measures the amount of pores whose radius is smaller than $R$. By definition, the pore size distribution $f$ ( $r$ ) can be obtained by the cumulative function by derivation:

$$
f(r)=\frac{d F(r)}{d r}
$$

Figure $1 \mathrm{a}$ and $\mathrm{b}$ allows visualising the link between the cumulative function $F(R)$ and the pore size distribution $f(r)$. The use of the logarithmic scale for the pore radius $r$ is justified by its wide range of variation. Figure 1a shows an example of cumulative function $F(R)$, giving a value $F(2 \mu \mathrm{m})=0.865$ for a pore radius equal to $2 \mu \mathrm{m}$, which indicates that $86.5 \%$ of the pore volume is made of pores having a radius smaller than $2 \mu \mathrm{m}$. Figure $1 \mathrm{~b}$ shows the same information in terms of pore size distribution $f(r)$ : the integral over $f(r)$ up to $2 \mu \mathrm{m}$ is equal to 0.865 . With reference to this example, it is worth noting that, despite the fact that the value of $f\left(10^{-9} \mathrm{~m}\right)$ is significantly greater than zero, the contribution of pores smaller than $10^{-9} \mathrm{~m}$ to the total pore volume is negligible. This observation justifies the introduction of the PSD function as an alternative variable to represent the distribution of the pore sizes. With

$$
F^{e}(R)=e \cdot F(R)
$$

representing the contribution of the pores having a size smaller than $R$ to the total void ratio, $e$, the PSD function is defined as

$$
P S D(r)=\frac{d F^{e}(r)}{d(\log (r))}=e \cdot \frac{d F(r)}{d r} \cdot r \cdot \ln (10)=e \cdot f(r) \cdot r \cdot \ln (10)
$$

In Figure 1c, the PSD derived from the $F(R)$ shown in Figure 1a highlights how the contribution of the different pore radii to the total void ratio can be more effectively visualised. Following the previous definition, the integral over the PSD function up to $2 \mu \mathrm{m}$ corresponds to the $86.5 \%$ of the total void ratio of the sample.

\subsection{Single-porosity media}

According to the framework introduced by Millington and Quirk [36] and Mualem [37], as a first approximation the pores in the soil can be represented by a bundle of different sized cylindrical tubes. Under this assumption, which disregards the connectivity between the pores, during a wetting process the different classes of pores are sequentially filled starting from the smallest ones. This allows identifying $F(R)$ with the current degree of saturation $\operatorname{Sr}(R)$ :

$$
\operatorname{Sr}(R)=F(R)=\int_{R \min }^{R} f(r) d r
$$

and $F^{e}(R)$ with the current water ratio $e_{w}(R)$, defined as the volume of filled pores with respect to the volume of the solids:

$$
e_{w}(R)=\operatorname{Sr}(R) \cdot e=F^{e}(R)
$$



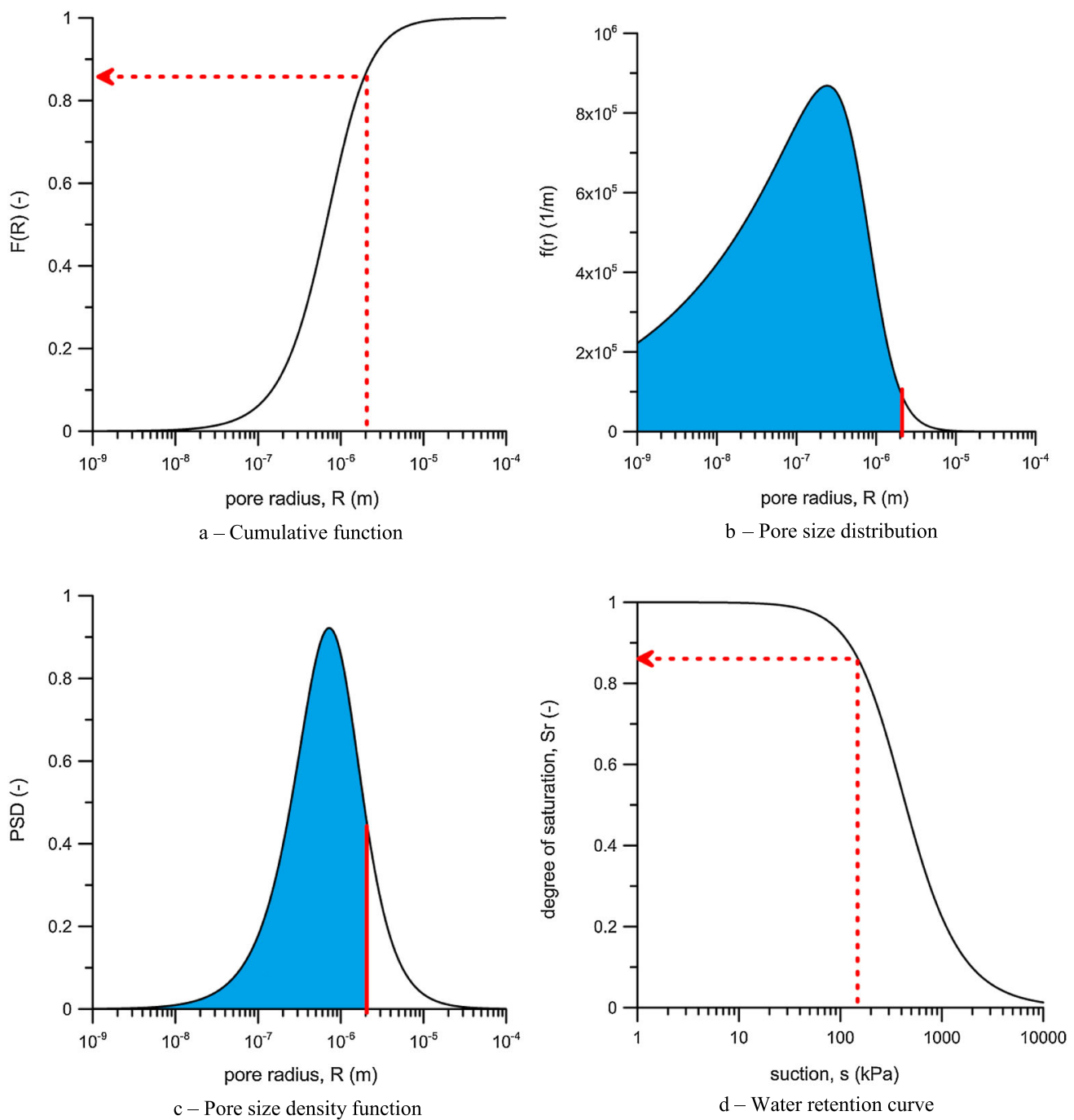

Figure 1. Conceptual link between cumulative function, pore size distribution, pore size density function and water retention curve.

The Laplace equation

$$
\psi(r)=\frac{2 T \cos \alpha}{r}=\frac{C}{r}
$$

allows to associate a value of suction $\psi$ to each pore radius $r$. In Equation (9), $T$ is the surface tension of the pore fluid, and $\alpha$ is the contact angle between the fluid and the soil particles. The quantity $C=2 T \cos \alpha$ is constant for a given soil mineralogy and chemical composition of the pore fluid; therefore,

$$
\operatorname{Sr}(R)=\int_{R \min }^{R} f(r) d r=\int_{R \min }^{R} f\left(\frac{C}{\psi(r)}\right) d r=G(\psi)
$$

The relationship $S r=G(\psi)$ is generally called water retention curve and is represented in Figure 1d. The latter conceptually coincides with Figure 1a, where pore radius is substituted with suction. The 
comparison shows that, in this example, a degree of saturation equal to 0.865 is predicted for a suction of $146 \mathrm{kPa}$, which is the value corresponding to a pore radius of $2 \mu \mathrm{m}$ according to the Laplace equation.

It is worth noting that the conceptual link between the pore size distribution and the water retention curve has a general value, although different functions $\psi(r)$ could be introduced to more accurately reproduce the retention mechanisms for different pore shapes [32]. According to the previous equations, a one-to-one correspondence between a chosen water retention curve model and a pore size distribution can be obtained. A convenient simple expression for the pore size distribution can be formulated starting from the most common equation used for water retention curve models, that is, the van Genuchten equation [2]:

$$
G(\psi)=\operatorname{Sr}(\psi)=\left[\frac{1}{1+(\alpha \psi)^{n}}\right]^{m}
$$

where $\alpha, m$ and $n$ are three model parameters. The effects of the three parameters $\alpha, m$ and $n$ on the water retention curve shape are well known and are discussed, for example, by Fredlund and Xing [3]. The analytical expressions for $F(R), f(R)$ and PSD associated to the van Genuchten model are presented in Appendix A.

\subsection{Double-porosity media}

Clays compacted on the dry side of the Proctor plane, up to the optimum water content, are generally characterised by a bimodal pore size distribution. For such materials, pores can be classified into two main classes, intra-aggregate pores (micro-pores) and inter-aggregate pores (macro-pores), and the cumulative pore size distribution can be obtained as the superposition of the cumulative distributions of the two classes of pores. Following the framework introduced in the previous section, the total degree of saturation of a soil composed by two classes of pores can be defined as

$$
S r=\frac{\phi_{m}}{\phi} S r^{m}+\frac{\phi_{M}}{\phi} S r^{M}
$$

where, $\phi_{m}$ is the porosity of the microstructure $\left(V_{v m} / V_{t o t}\right), \phi_{M}$ is the porosity of the macrostructure $\left(V_{v M} / V_{t o t}\right)$, and $\phi$ is the total porosity with $V_{w m}$ and $V_{w M}$ being the volume of water in the micropores and the macro-pores respectively, and $V_{v m}$ and $V_{v M}$ are the corresponding volume of voids.

The cumulative distribution $F(R)$ now reads

$$
F(R)=\int_{R \min }^{R} f(r) d r=\operatorname{Sr}(R)=\frac{\phi_{m}}{\phi} \operatorname{Sr}^{m}(R)+\frac{\phi_{M}}{\phi} S r^{M}(R)
$$

and, by definition, the pore size distribution $f(r)$ can be calculated as

$$
f(r)=\frac{\phi_{m}}{\phi} \frac{d S r^{m}(r)}{d r}+\frac{\phi_{M}}{\phi} \frac{d S r^{M}(r)}{d r}
$$

Two water retention functions $S r_{m, M}=G^{m, M}(\psi)$ must be defined for the two domains. If the van Genuchten equation is adopted for each domain, the two functions can be written as

$$
\begin{aligned}
& S_{r m}=\frac{V_{w m}}{V_{v m}}=\left[\frac{1}{1+\left(\alpha_{m} \Psi\right)^{n_{m}}}\right]^{m_{m}} \\
& S_{r M}=\frac{V_{w M}}{V_{v M}}=\left[\frac{1}{1+\left(\alpha_{M} \Psi\right)^{n_{M}}}\right]^{m_{M}}
\end{aligned}
$$

where $n_{m}, m_{m}, \alpha_{m}$ and $n_{M}, m_{M}, \alpha_{M}$ are the van Genuchten parameters for the micro-pores and the macro-pores, respectively. The complete set of equations for the corresponding bimodal water 
retention model and the associated PSD function has been discussed by Dieudonne et al. [35], and it is reported in Appendix A for the sake of completeness. An example of a bimodal PSD curve, which can be simulated with the framework proposed, is shown in Figure 2a, which clarifies the link between the global PSD curve and its micro and macro components. It is worth noting that, given that the two distributions overlap, some pore radii can belong to both the intra-aggregate and the inter-aggregate regions.

By means of the Laplace equation, the water retention curve in Figure $2 b$ and $c$ is obtained: for a porous medium whose PSD is bimodal, the retention curve is thus obtained by the superposition of two distinct retention mechanisms, one for the microstructure and one for the macrostructure, whose envelope results in a final bimodal water retention curve. Here, and in the following, we assume the different structure levels always to be in thermodynamical equilibrium, hence the current value of suction to apply to both the microstructure and the macrostructure levels. Each domain is characterised by its own air-entry value: in the example provided in Figure $2 b$, for a suction $s=100 \mathrm{kPa}$, the degree of saturation of the macro-pores is lower than 0.2 , whilst for the same suction value, the micro-voids are still saturated $\left(S r_{m}=1\right)$.

It is worth noting that the degree of saturation is not additive, as it is obtained by the sum of the micro and macro degree of saturation, weighted by the corresponding volumetric fractions (Equation (14)). On the contrary, the water ratio, $e_{w}$, defined as the volume of water over the volume of solids, is additive and can be calculated by summing its micro and macro fractions: $e_{w}=e_{w m}+e_{w M}$ (Figure 2c).

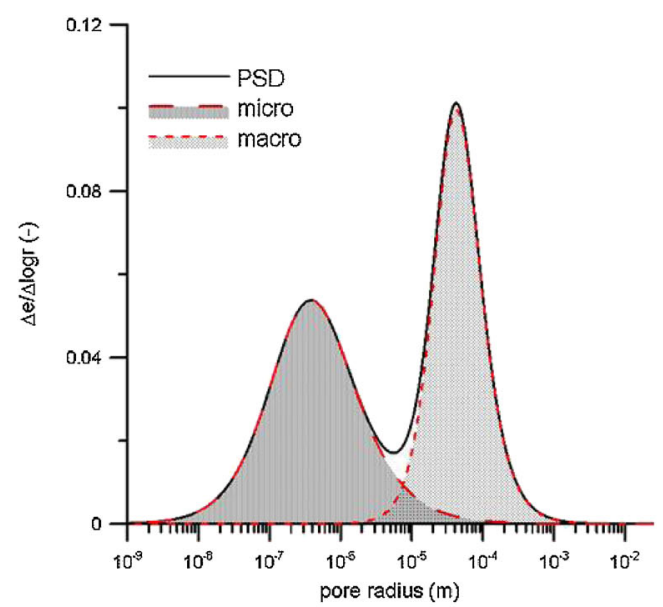

$\mathrm{a}-$ Pore size density function

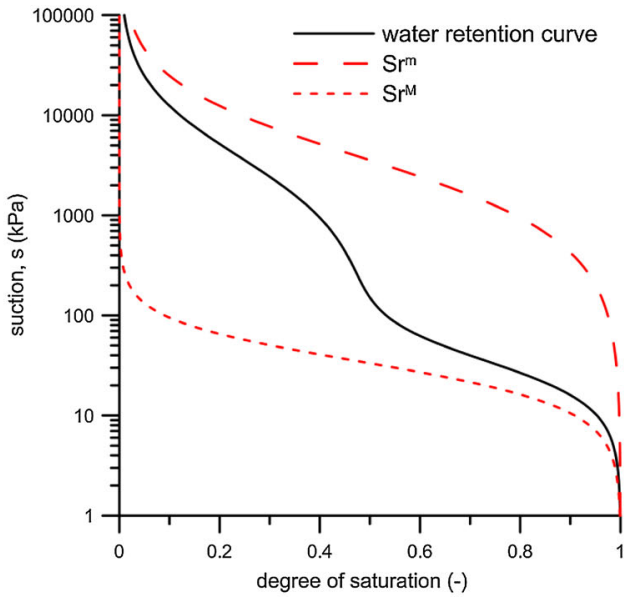

$b-$ Water retention curve in terms of degree of saturation

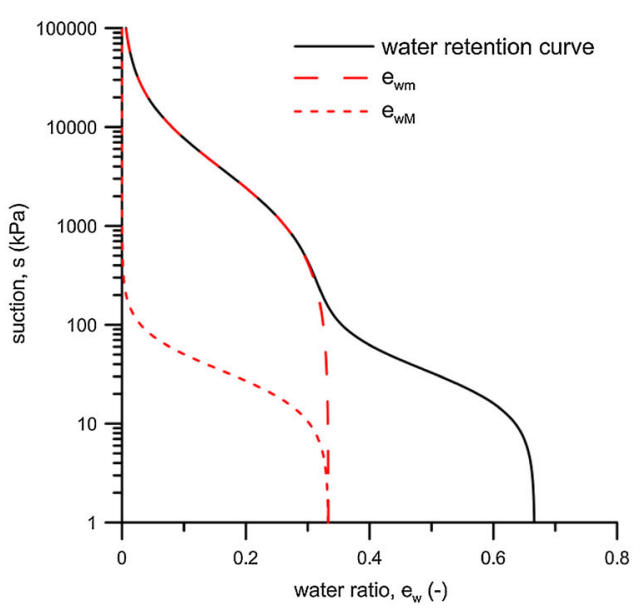

$c-$ Water retention curve in terms of water ratio

Figure 2. Conceptual link between PSD and water retention curve for a double-porosity medium. 


\section{THE ROLE OF PORE SIZE DENSITY EVOLUTION IN MODELLING WATER RETENTION PROCESSES}

Despite the as-compacted double-porosity fabric of compacted clays, soil water retention curves obtained in the laboratory for these materials rarely show a bimodal shape. This seeming paradox can be explained if the fabric evolution along hydro-mechanical paths is taken into account. In order to obtain a physically based link between the PSD function and the water retention curve determined by means of experimental tests, a number of PSD data were analysed on compacted clays subjected to different hydro-mechanical paths. MIP data are exploited to this aim. For the sake of simplicity, hysteresis is not addressed here, and reference is equally made to drying or to wetting paths.

The effects of mechanical paths are discussed firstly. Experimental data involving different pore size distributions of compacted bentonite-sand mixture samples at different dry densities and at the same water content are presented in Figure 3a [38, 39]. Bentonite-sand samples (70-30\% proportion on dry mass basis) were compacted at a water content between $10 \%$ and $11 \%$ and at dry densities of $1.67 \mathrm{Mg} / \mathrm{m}^{3}\left(e_{0}=0.62\right), 1.80 \mathrm{Mg} / \mathrm{m}^{3}\left(e_{0}=0.5\right)$ and $1.97 \mathrm{Mg} / \mathrm{m}^{3}\left(e_{0}=0.37\right)$. Increasing the compaction effort at constant water content shifts the peak of the macro-pore distribution towards smaller pore radii, and it reduces its height, according to $\mathrm{Hu}$ et al. [34]. On the contrary, the microstructure void distribution is hardly affected by the compaction density. Similar results have been observed on different compacted clayey soils, such as Boom clay [30, 33], London clay [23], FoCa clay [40], Speswhite kaolin [41] and Barcelona silty clay [42].

MIP data are used here to derive a theoretical matric suction-water ratio relationship, following Romero and Simms [43], who suggested that the mercury intrusion path can be assimilated to the air intrusion along the desorption path of water retention curve. Theoretical water retention curves corresponding to the frozen initial fabric obtained upon compaction from the PSD data of a bentonite-sand mixture are shown in Figure 3b, which shows the evidence of the dominant role of dry density on the air-entry value of the macro-pores.

The effect of hydration processes at different mechanical constraints is highlighted in Figure 4a. With reference to compacted Boom clay, the PSD of the as-compacted material is compared with the PSDs of the following: (i) a constant volume-saturated sample (isochoric saturation); (ii) a sample saturated at a constant vertical stress $\sigma_{v}$ of $85 \mathrm{kPa}$; and (iii) a sample saturated at a constant vertical stress of $600 \mathrm{kPa}$. All the saturated samples, subjected to freeze drying before MIP test in order to preserve the actual final fabric of the material, show a dominant unimodal PSD function. An interpretation of these results is provided by Romero et al. [30]: the isochoric and the $\sigma_{v}=85 \mathrm{kPa}$ saturation paths affect both inter-aggregate and intra-aggregate pores. After saturation,

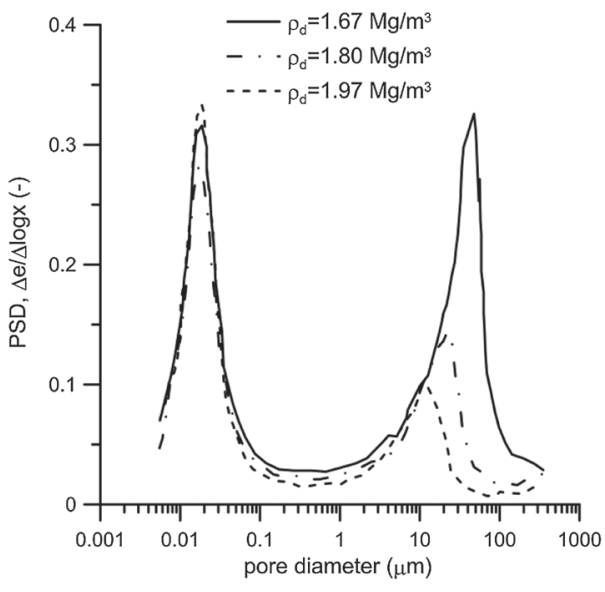

a - PSD at different compaction density

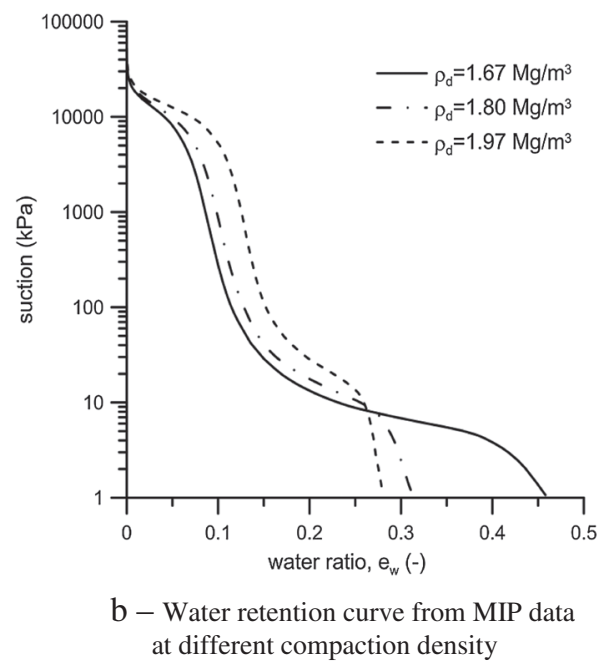

Figure 3. Compacted bentonite-sand mixture at different void ratios (same water content), from Wang et al. [38] and Saba et al. [39]. 

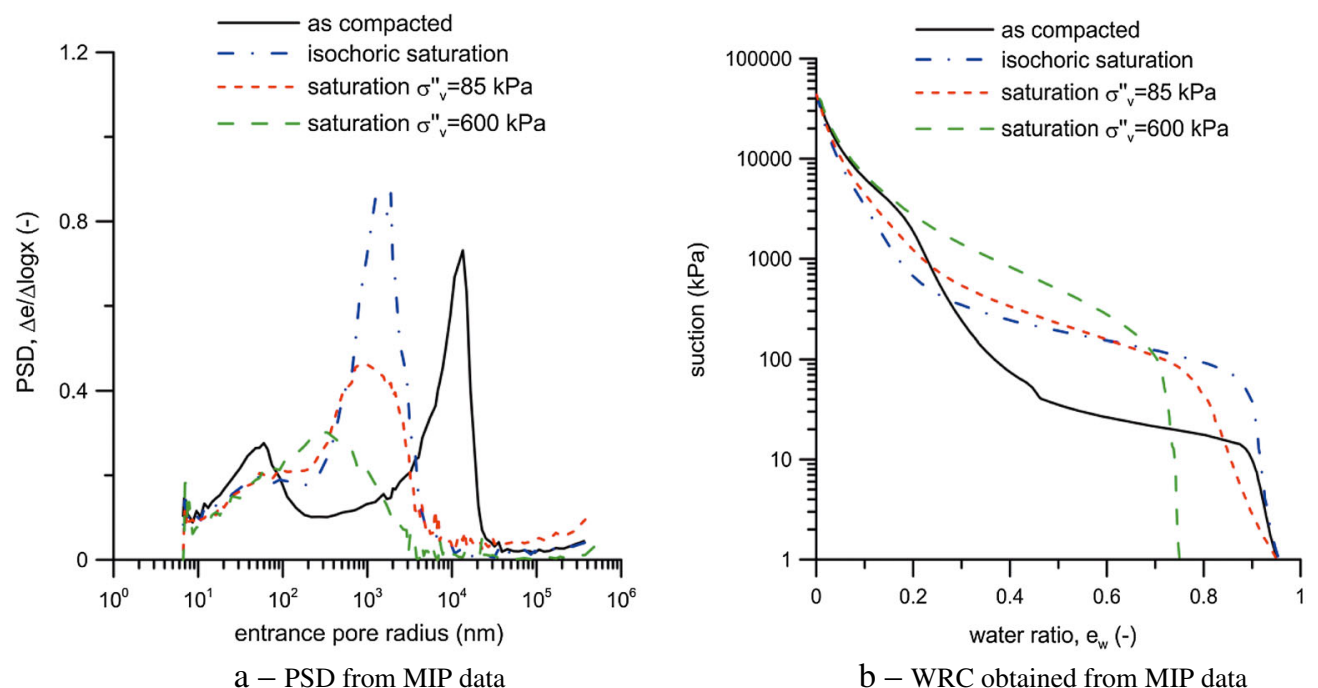

Figure 4. Compacted Boom clay samples saturated with different mechanical constraints, from Della Vecchia [22] and Romero et al. [30].

the two samples display a similar fabric dominated by a single peak between 1 and $2 \mu \mathrm{m}$, which is originated by both expansion of the micro-pores and invasion of macro-pores. A more significant reduction of the inter-aggregate porosity is observed for the sample saturated at $\sigma_{v}=600 \mathrm{kPa}$, because of the additional effect of macro-pore collapse upon wetting at high stress level (as discussed in [33]). Similar results have been presented by Lloret and Villar [44] for compacted FEBEX bentonite samples, whilst filling of the macro-porosity due to expanding microstructure has been highlighted by Cui et al. [45].

Theoretical water retention curves obtained from MIP data corresponding to the final fabric are presented in Figure 4b. According to the PSD data, the bimodal water retention curve of the ascompacted state turns into a unimodal curve after saturation. The role of void ratio is again evident in the macroscopic part of the curve, essentially in terms of air-entry value: the highest air-entry value is found for the sample that experienced largest plastic compression strain upon wetting $\left(\sigma_{v}=600 \mathrm{kPa}\right)$. Besides, the comparison between the curves representing the as-compacted sample and the isochoric saturated one testifies that, at the same void ratio, the air-entry value is a function of the current water content. Actually, as the water content increases, the aggregates swell, reduce the volume of the macro-pores and increase the air-entry value. The role of aggregate swelling is evident also noting the significant differences between the as-compacted sample and the saturated ones in the micro-porosity domain. Increasing the aggregate size implies not only an increase in the air-entry value of the macro-pores but also a reduction of the air-entry value of the micro-pores.

Notwithstanding the consistent pattern of information provided on water retention properties by MIP data, in the practice, a water retention model cannot be obtained directly by derivation of the resulting PSD. On the one hand, the derivation of the matric suction-degree of saturation relationship, by means of relationship such as the Laplace law, should be limited to the low suction range (usually lower than $2 \mathrm{MPa}$ ), in which capillary mechanisms dominate. However, Prapaharan et al. [46] and Romero et al. [19] have shown that, also in this range, MIP predictions substantially underestimated water contents at suction greater than tens of kiloPascal. Amongst other reasons, Romero and Simms [43] attributed this discrepancy to different accessibilities of pores in MIP and retention curve samples. On the other hand, the fabric of soils changes continuously during drying and wetting paths, as highlighted amongst others by Simms and Yanful [47, 29], who showed the PSD evolution during water retention testing. As a consequence, the retention properties predicted from a single PSD cannot capture the material behaviour at different suction levels: a single PSD could only predict the retention properties that the soil would show if its fabric were frozen at the hydraulic state at which the pore size distribution was determined. Therefore, numerous PSDs are required to determine a complete retention curve [30, 15, 32]. Nonetheless, despite the fact that the 
parameters of a water retention model cannot be directly calibrated on MIP data, the quantitative information on pore size distribution can provide information on the constitutive laws for the aggregate deformation, as well as on the form of the evolution laws for the parameters of the water retention curve to properly take into account the related PSD evolution.

To analyse the evolution of the retention properties along different hydro-mechanical paths, a calibration procedure was evaluated directly on the experimental data obtained by MIP tests. The first step is the choice of a suitable analytical model for the water retention behaviour, ideally one for each structural domain. Given the analytical expression for the water retention curve, the analytical expression of the corresponding pore size distribution can be obtained following the procedure introduced in the previous section. The chosen analytical PSD model can be calibrated by best fitting of the experimental MIP data, keeping fixed the greatest number of parameters as possible and correlating the non-constant parameters with hydro-mechanical state variables.

The approach is shown by analysing the results on different compacted clay soils, with reference to the model presented by Dieudonné et al. [35] and reported in Appendix A. The calibration procedure was performed on compacted Boom clay, as well as on compacted London clay [23], MX-80 clay [48], Hong Kong clay [49] and bentonite-sand mixture [38, 39]. The law ruling the evolution of microstructural void ratio $e_{m}$ along generalised hydro-mechanical paths can also be directly established during the calibration phase. Good correlation was found between the void ratio of micro-voids, $e_{m}=V v_{m} / V_{s}$, and the water ratio, $e_{w}$, which can be given the form:

$$
e_{m}=\beta_{0} e_{w}^{2}+\beta_{1} e_{w}+e_{m, 0}
$$

where $\beta_{0}$ and $\beta_{1}$ are parameters that quantify the swelling potential of the aggregates and $e_{m, 0}$ is the microstructural void ratio for the dry material $\left(e_{w}=0\right)$. In a reasonable water ratio range, Equation (15) provides predictions similar to those of the bilinear expression proposed by Romero et al. [30], whilst providing a continuous evolution of $e_{m}$ with the water ratio.

As a conceptual difference from the framework proposed by Della Vecchia [22] and Romero et al. [30], MIP data are directly used to calibrate the pore size distribution model by means of Equation (15), without any a priori definition of a discriminating diameter between micro-pore and macro-pore size domains. According to Romero et al. [30], the tendency of the aggregates to swell appears to be linked to the plasticity of clayey soils, and the microstructural void ratio at the dry state can be reasonably considered a function of the mineralogical properties of the clayey fraction. Romero et al. [30] also proposed a correlation between the microstructural void ratio corresponding to dry macrostructure and saturated micro-pores, $e^{*}{ }_{m}$, and the specific surface of the clayey fraction. In order to improve the practical utility of the framework proposed, further correlations are here evaluated with material properties easy to obtain in standard soil mechanics laboratories. These correlations, reported in Figure 5, have been obtained analysing the PSD evolution of the selected clayey soils from MIP data. Figure $5 \mathrm{a}$ and $\mathrm{b}$ shows possible correlations that link the parameters $\beta_{0}$ and $\beta_{1}$ to the activity index of the material, defined as the ratio between the plastic index and the percentage of clay-sized particles. As for the parameter $e_{m 0}$, a relation similar to the one proposed by Romero et al. [30] for $e^{*}{ }_{m}$ should be valid. However, because of the possible dependence of the initial microstructural void ratio on compaction energy, compaction density and compaction water content, no satisfying correlation for $e_{m 0}$ could be obtained with this data set.

Figure 6 shows the results of the calibration procedure for the parameter $1 / \alpha_{m}$, that is, the air-entry value of the intra-aggregate porosity, as a function of the current intra-aggregate void ratio, $e_{m}$, for compacted Boom clay, London clay and Hong Kong clay. The evolution of the air-entry value of the micro-pores seems to be well normalised with the microstructural void radio $e_{m}$, by means of the same interpolation function. An exponential fitting law linking $\alpha_{m}$ to $e_{m}$ can thus be proposed:

$$
\frac{1}{\alpha_{m}}=\alpha_{1}^{(m)} \exp \left(-\alpha_{2}^{(m)} e_{m}\right)
$$

where $\alpha_{1}^{(m)}$ and $\alpha_{2}^{(m)}$ are model parameters. Equation (16) has a clear physical meaning, suggesting that the greater the size of the intra-aggregate pores, the lower the air-entry value of the aggregates. For the 

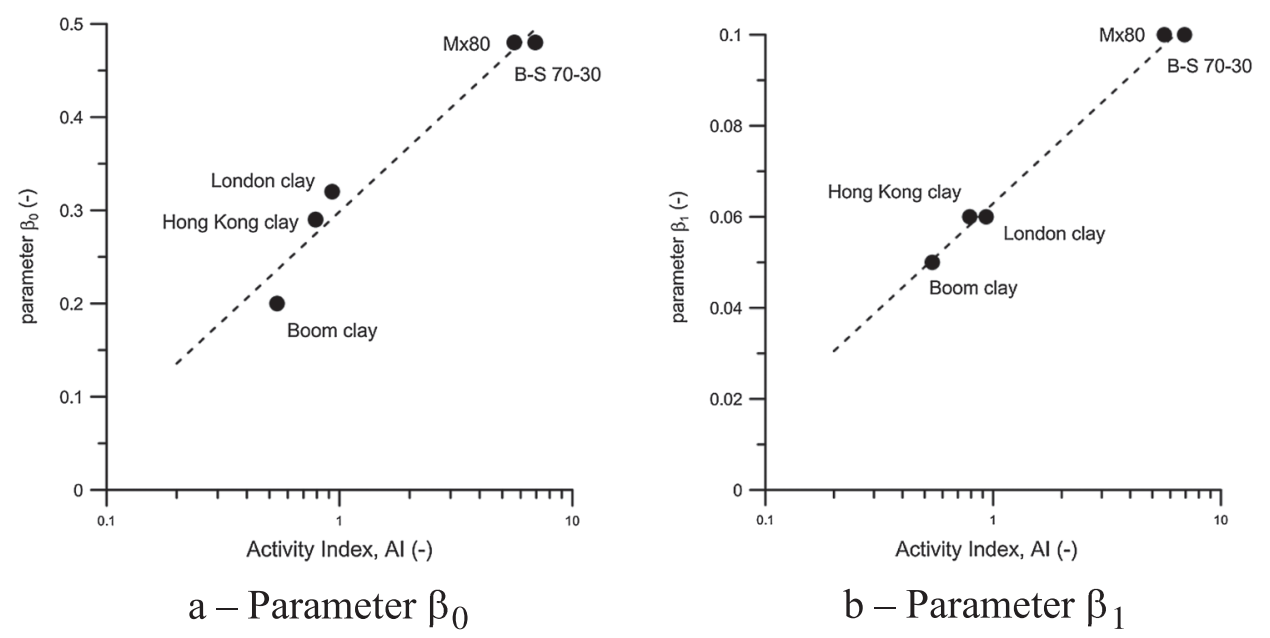

Figure 5. Correlations for the swelling properties of the aggregates. Experimental data from Della Vecchia [22] and Romero et al. [30] for Boom clay, Monroy et al. [23] for London clay, Li and Zhang [49] for Hong Kong clay, Wang et al. [38] and Saba et al. [39] for bentonite-sand (S-B 70-30), and Delage et al. [48] and Wang [59] for MX-80.

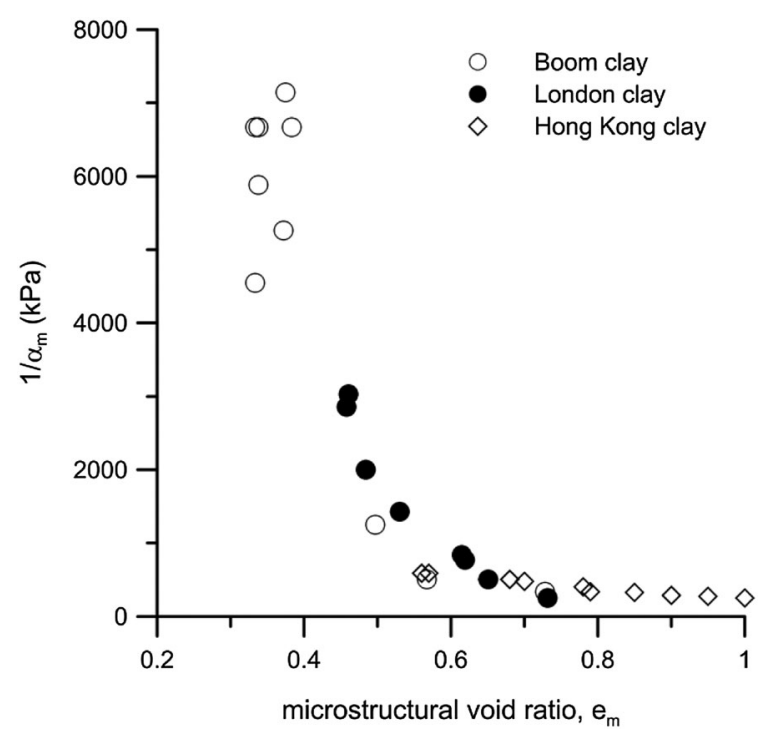

Figure 6. Evolution of $\alpha_{m}$ for three low-medium activity clays. Experimental data from: Della Vecchia [22] and Romero et al. [30] for Boom clay, Monroy et al. [23] for London clay, and Li and Zhang [49] for Hong Kong clay.

three materials analysed, the relationship between $\alpha_{m}$ and $e_{m}$ seems unique. However, the three clays considered are characterised by comparable activity values; hence, no general validity is claimed for the proposed relationship, and more experimental data are needed to extend this conclusion to other materials with significantly different mineralogical properties.

As for the results of the calibration procedure for the evolution of $1 / \alpha_{M}$, that is, the air-entry value of the macrostructure, good correlation was found with the ratio of inter-aggregate pore volume over the total volume of pores, $e_{M} / e$, as well as with the normalised inter-aggregate void ratio $e^{*}{ }_{M}=V_{v M} /\left(V_{s}+V_{v m}\right)$, as proposed by Mašín [50]. Figure 7 shows the calibrated values of $1 / \alpha_{M}$ for compacted Boom clay, Honk Hong clay, London clay and bentonite-sand 70-30, together with an exponential trend for each material. Differently from Figure 6, it is not possible to recognise a unique trend for all these materials, probably because of different inter-aggregate fabrics imparted by the different 
compaction processes. However, it is possible to obtain a unique evolution law for $\alpha_{M}$, to be calibrated on each material:

$$
\frac{1}{\alpha_{M}}=\alpha_{1}^{(M)} \exp \left(-\alpha_{2}^{(M)} \frac{e_{M}}{e}\right)
$$

where $\alpha_{1}^{(M)}$ and $\alpha_{2}^{(M)}$ are model parameters. The trends obtained seem consistent with the phenomenological power law proposed by Gallipoli et al. [8], Tarantino and De Col [41] and Gallipoli [14], which link the air-entry value to the void ratio. However, in this case, not only the effect of different dry densities of the material is taken into account to rule the evolution of $\alpha_{M}$, but also the size of the evolving aggregates, according to the double-porosity nature of compacted clays.

\section{THEORETICAL PREDICTIONS OF THE FRAMEWORK}

The previous framework provides a physically based link between the retention properties and the state variables at the microscopic and at the phenomenological levels, and it also helps in understanding and visualising some peculiar aspects of the retention behaviour of compacted clayey soils. As introduced in the previous section, experimental data of compacted clayey soils clearly show that the water retention curve shows a unimodal response during direct measurement, despite the as-compacted bimodal pore size distribution. This evidence is a consequence of the evolution of soil fabric during the hydro-mechanical path followed during the measurement of the water retention domain. The interpretation provided by the framework is shown in Figure 8. Let us consider a porous medium with an initially bimodal pore size distribution (Figure 8a, continuous line, $e_{w 0}=0.4, e_{0}=0.9$ ), subjected to a wetting process at constant volume: this path can be associated with the determination of the wetting branch of a water retention curve for a given void ratio. During hydration (from $e_{w}=0.4-0.6$ ), the aggregates expand, and partially fill the macro-porosity due to the constant volume constraint $\left(\Delta e=\Delta e_{m}+\Delta e_{M}=0\right.$ implies $\left.\Delta e_{M}=-\Delta e_{m}\right)$. As a consequence, the inter-aggregate void ratio reduces, and the intra-aggregate void ratio increases (Figure 8a, dotted line). According to the correlations proposed in Figures 6 and 7 , an increase in the air-entry value of the

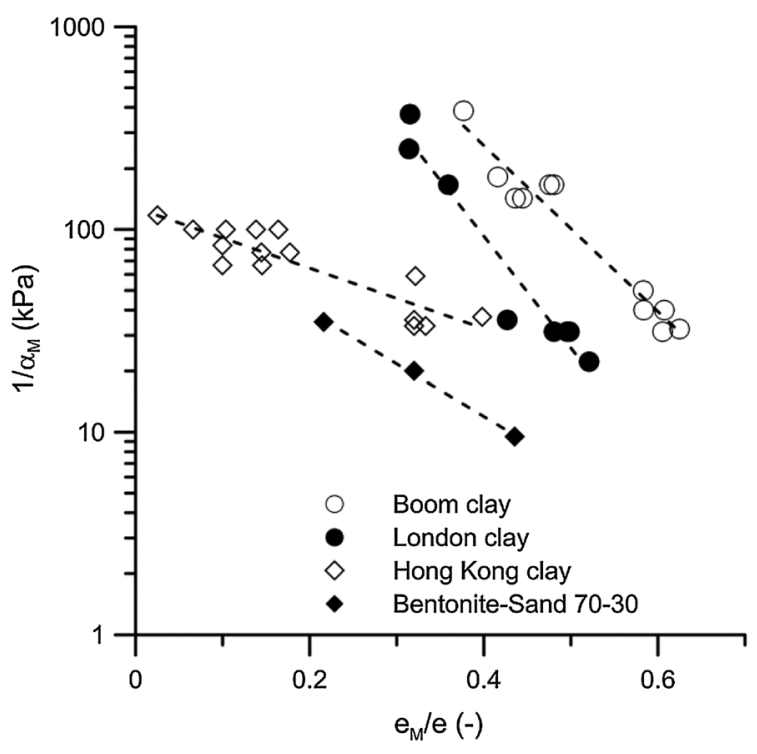

Figure 7. Evolution of $\alpha_{M}$ with the ratio $e_{M} / e$. Experimental data from: Della Vecchia [22] and Romero et al. [30] for Boom clay, Monroy et al. [23] for London clay, Li and Zhang [49] for Hong Kong clay, and Wang et al. [38] and Saba et al. [39] for bentonite-sand (B-S 70-30). 


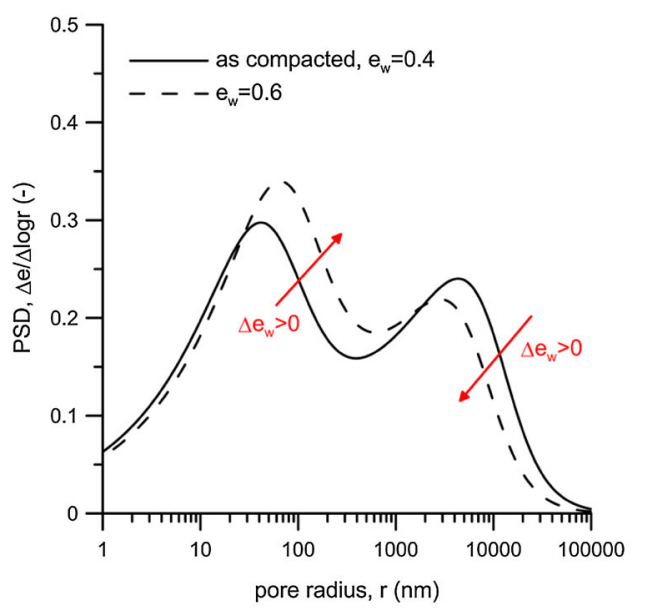

a - PSD evolution due to wetting at constant volume

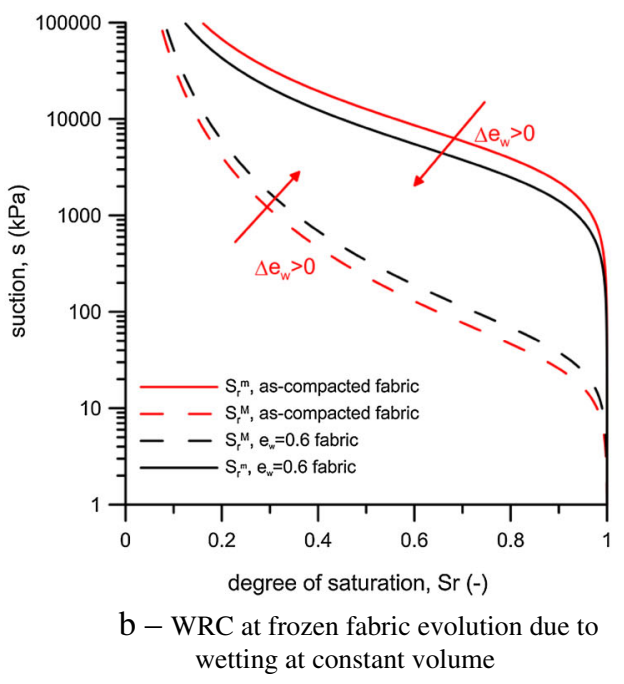

Figure 8. Theoretical predictions of the framework along wetting at constant volume.

macrostructure (i.e. decrease of $\alpha_{M}$ ) and a reduction of the air-entry value of the microstructure (i.e. increase of $\alpha_{m}$ ) are expected, as shown in Figure 8b. The dotted lines show the water retention curve of the macro-pores for two different fabrics, corresponding to the as-compacted state $\left(e_{w}=0.4\right)$ and to the state of the sample after wetting $\left(e_{w}=0.6\right)$ : an increase in water content is associated to an increase of the air-entry value of the macro-pores. On the contrary, continuous lines show that the air-entry value of the micro-pores reduces as a consequence of wetting.

As the water content increases, the peak of the macroscopic PSD moves leftwards. Vice versa, the aggregates expand, and their pore size distribution moves towards larger radii, finally ending in a global unimodal PSD: in this state, the micro-porosity and the macro-porosity are significantly superimposed (Figure 9a). As a consequence, the air-entry values of the water retention curves of the micro-pores and of the macro-pores tend to the same value, leading to an apparent unimodal water retention curve (Figure 9b).

As the fabric of the material is never frozen in a given state but continuously evolves along hydration, none of the plotted retention curves at different frozen states correspond to the measured water retention curve, which is actually a subset of points on the curves corresponding to the current different soil fabrics, as discussed by Simms and Yanful [29], Romero et al. [30], Zhou et al. [15] and Beckett and Augarde [32]. Figure 10 shows the water retention curves, obtained by the PSD functions corresponding to different frozen fabrics, and the water retention curve predicted by the model summarised in Appendix A, which is characterised by a micro-fabric evolution ruled by Equations (16) and (17). The water retention curve for evolving fabric shows in the low suction range a hydraulic behaviour similar to the one of the saturated material. At increasing suction, the predicted curve crosses the 'frozen' curves corresponding to different water ratios, reaching the curve corresponding to the as-compacted state for a suction of the order of $2 \mathrm{MPa}$. For higher suctions, the evolving curve is quite similar to the as-compacted one, because of the limited aggregate deformation in the high suction range [30, 17].

By tracking the explicit dependence of water retention on PSD evolution along different hydro-mechanical paths, the proposed framework is able to take into account the variation in water retention properties obtained with different mechanical constraints. As evidenced by Loiseau et al. [51] and Cui et al. [52], the water retention curve under constant-volume and free-swell conditions can be significantly different. Figure 11a shows the model predictions in the two cases, starting from an as-compacted condition with $e_{w 0}=0.49, s_{0}=2 \mathrm{MPa}, e_{0}=0.9$ and reaching a final condition for the swelling sample with $e_{f}=1.2$. It is interesting to notice that these differences cannot just be explained in terms of void ratio variation, that is, different saturated water contents. The predicted water retention curves are evidenced in Figure 11b, where the higher air-entry value for the constant-volume retention curve can be appreciated. 


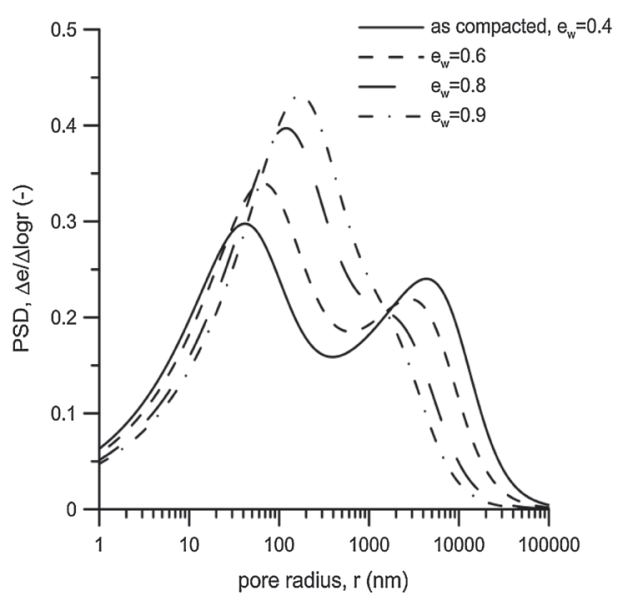

$\mathrm{a}-\mathrm{PSD}$ evolution upon hydration

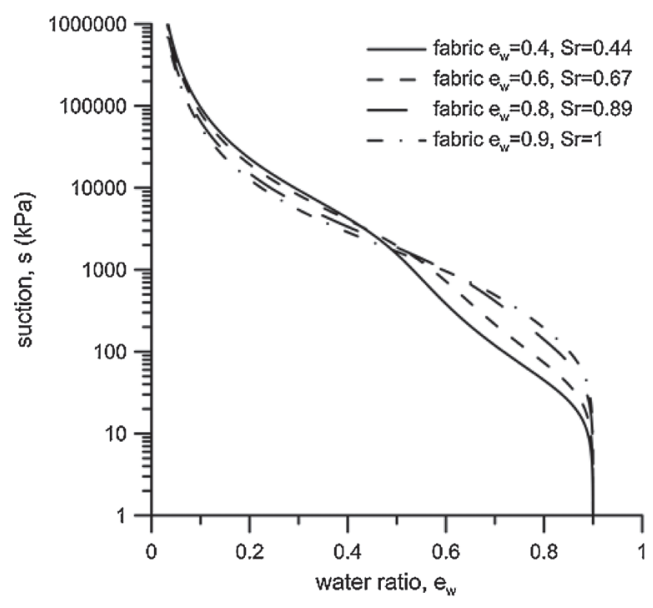

$\mathrm{b}-$ WRC evolution upon hydration

Figure 9. Continuous fabric evolution along saturation path.

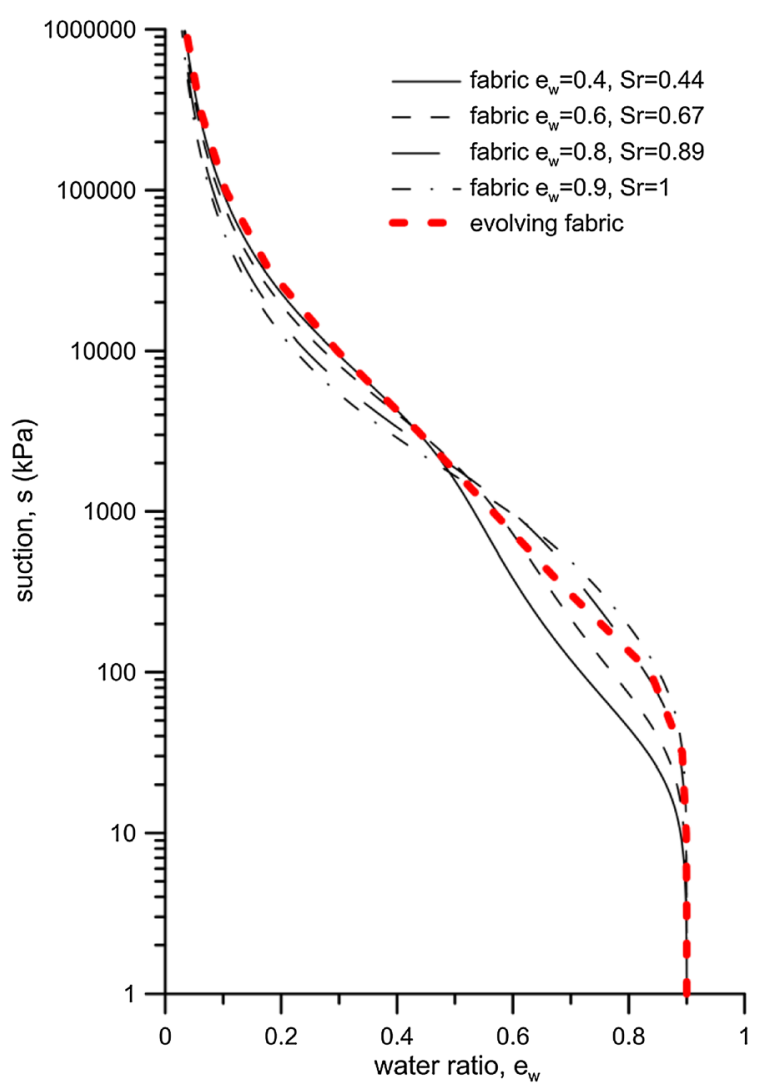

Figure 10. Water retention curve as the subset of the current states at evolving fabric.

The role of the micro-fabric along the two hydraulic paths is evident from Figure 11c, where the evolution of both $e_{m}$ and $e_{M}$ is shown for the two tests. As $e_{m}$ is proportional to $e_{w}$, the higher the water content of the sample, the largest the intra-aggregate void ratio. The simultaneous evolution of both total and microstructural void ratios implies significant difference in the macrostructural void ratio, and thus in the transport properties of the material as, for example, diffusivity [17] and hydraulic conductivity [53]. 


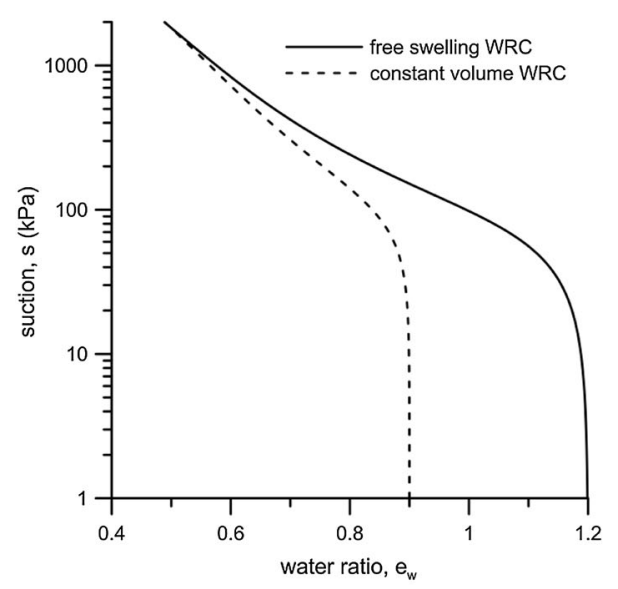

$\mathrm{a}-$ Water ratio vs. suction

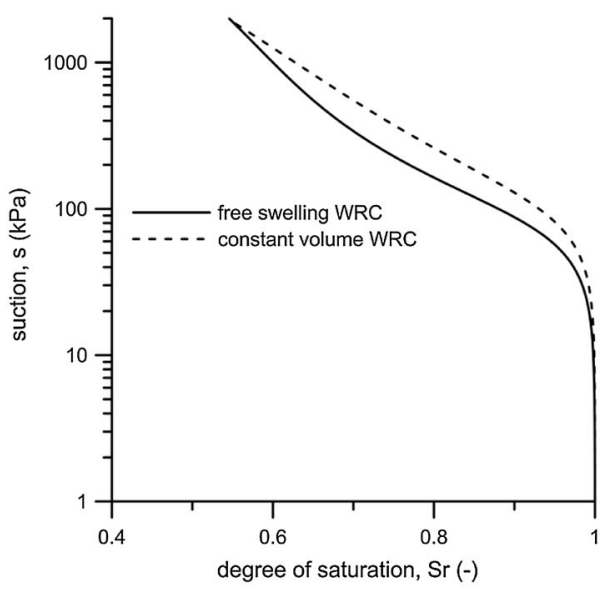

$\mathrm{b}-$ Degree of saturation vs. suction

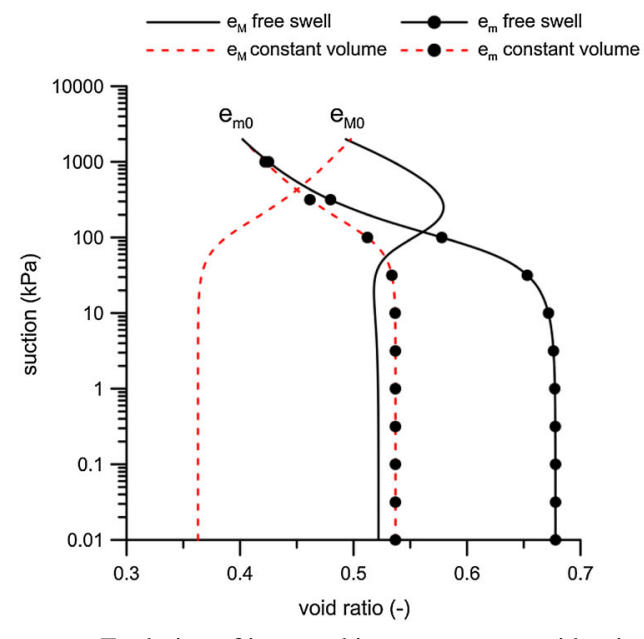

Figure 11. Water retention curves at constant-volume and free-swell conditions.

\section{VALIDATION OF THE WATER RETENTION MODEL}

The described approach was embedded in a double-porosity van Genuchten model (see Appendix A), which proved able to simulate the behaviour of double-porosity compacted soils. The hydromechanical response of compacted Boom clay was very well caught, as testified by the comparison in Figure 12 between experimental data of drying curves at constant void ratio and model predictions. Nonetheless, it has to be remarked that a huge body of MIP data was available to the aim of calibration for this soil [35]. For the approach to be used in the practice, at the laboratory or at the field scale, two subsequent steps are conceptually mandatory: (i) calibration should be possible without a need for dedicated microstructural tests and (ii) complex hydro-mechanical paths should be used for checking the modelling capabilities on different soils.

In a first attempt to validate the framework, the water retention of a compacted scaly clay (Palermo scaly clay) has been simulated, exploiting the analytical form of the correlations provided by MIP data to infer some of the parameters needed for calibration. Actually, the correlations proposed in Figure 5a $\left(\beta_{0}=0.2\right)$ and $\mathrm{b}\left(\beta_{1}=0.05\right)$ and in Figure $6\left(\alpha_{1 m}=180 \mathrm{MPa}, \alpha_{2}=9\right)$ were exploited for the microstructural contribution along the main drying branch. The remaining parameters were calibrated on the main drying and the main wetting branches of the water retention curve at a constant void ratio of $e=0.45$, presented by Airò Farulla $e t$ al. [54]. The results of the calibration procedure on the compacted scaly clay is shown in Figure 13a and b (dotted lines), together with the predictions (continuous lines) for the void ratios $e=0.50, e=0.55$ and $e=0.60$. 


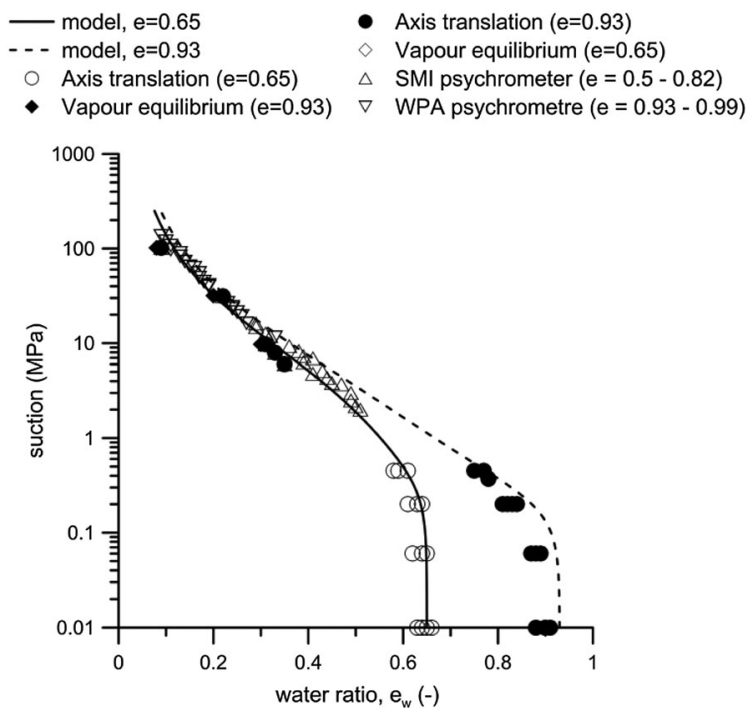

Figure 12. Main drying curves for compacted Boom clay at constant void ratio. Experimental data from Romero et al. [19] and Della Vecchia [22].
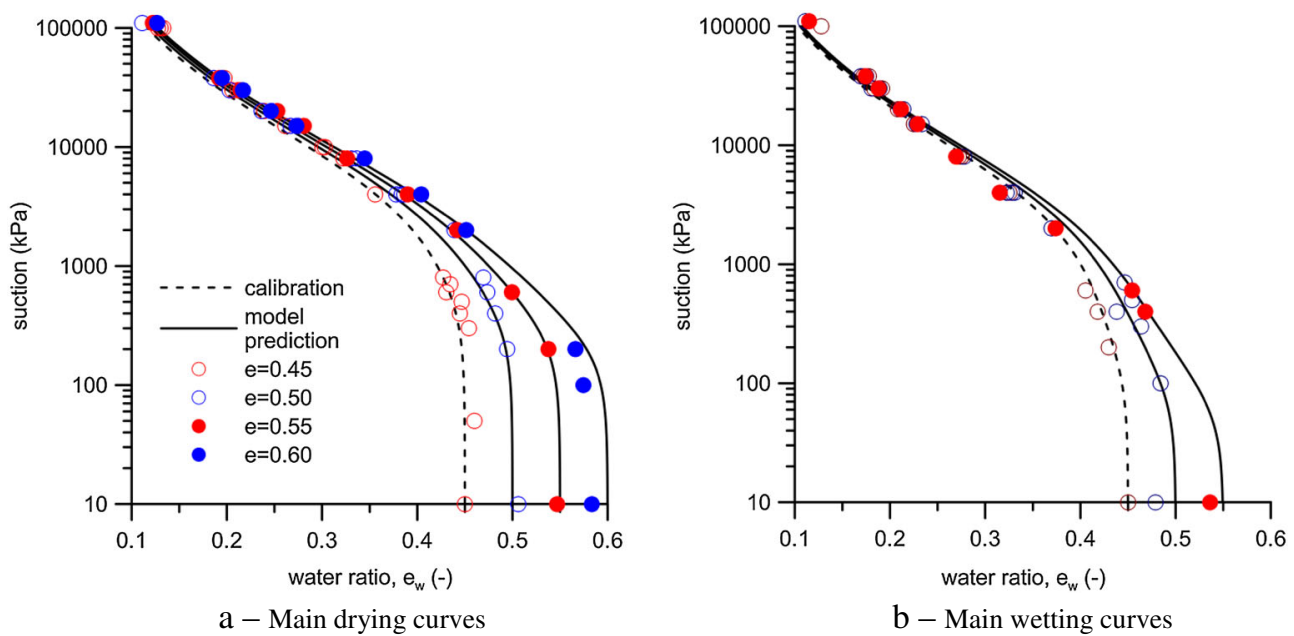

Figure 13. Water retention curve at constant void ratio for compacted scaly clay. Experimental data from Airò Farulla et al. [54].

A summary of the parameters obtained for the compacted scaly clay is presented in Table I. It is worth noting that by just changing $\alpha_{1 m}$ and $\alpha_{1 M}$, both the main drying and the main wetting could be simulated.

The performance of the calibrated model was evaluated in terms of water inflow/outflow predicted during cyclic suction changes and void ratio. The experimental data come from Airò Farulla [55], who simulated the changes in void ratio by means of the Barcelona expansive model $[56,57]$ in order to take into account the effects of the double porosity on the mechanical behaviour of the material. Experimental data were obtained on a sample statically compacted at initial dry unit weight of $17.3 \mathrm{kN} / \mathrm{m}^{3}$ and water content $w_{0}=15 \%$ (corresponding to a suction $s_{0}=800 \mathrm{kPa}$ ), then loaded up to $\sigma_{v}=800 \mathrm{kPa}$ and finally subjected to suction-controlled wetting-drying cycles. Experimental response of void ratio evolution during wetting-drying cycles is presented in Figure 14a, as a function of suction (Test A). The sample was then subjected to a loading step up to $1600 \mathrm{kPa}$, followed by unloading to $800 \mathrm{kPa}$ and again subjected to wetting-drying cycles (Test $\mathrm{B}$, Figure 14b). The last wetting-drying cycles were performed after unloading the sample to $\sigma_{v}=50 \mathrm{kPa}$ (Test C, Figure 14c). 
Table I. Water retention parameters for compacted scaly clay

\begin{tabular}{lcccccccc}
\hline & $n_{m}$ & $m_{m}$ & $\alpha_{1 m}(\mathrm{MPa})$ & $\alpha_{2 m}$ & $n_{M}$ & $m_{M}$ & $\alpha_{1 M}(\mathrm{MPa})$ & $\alpha_{2 M}$ \\
\hline Drying & 1.4 & 0.35 & 180 & 9 & 2 & 0.16 & 0.8 & 5 \\
Wetting & 1.4 & 0.35 & 150 & 9 & 2 & 0.16 & 0.2 & 5 \\
\hline
\end{tabular}
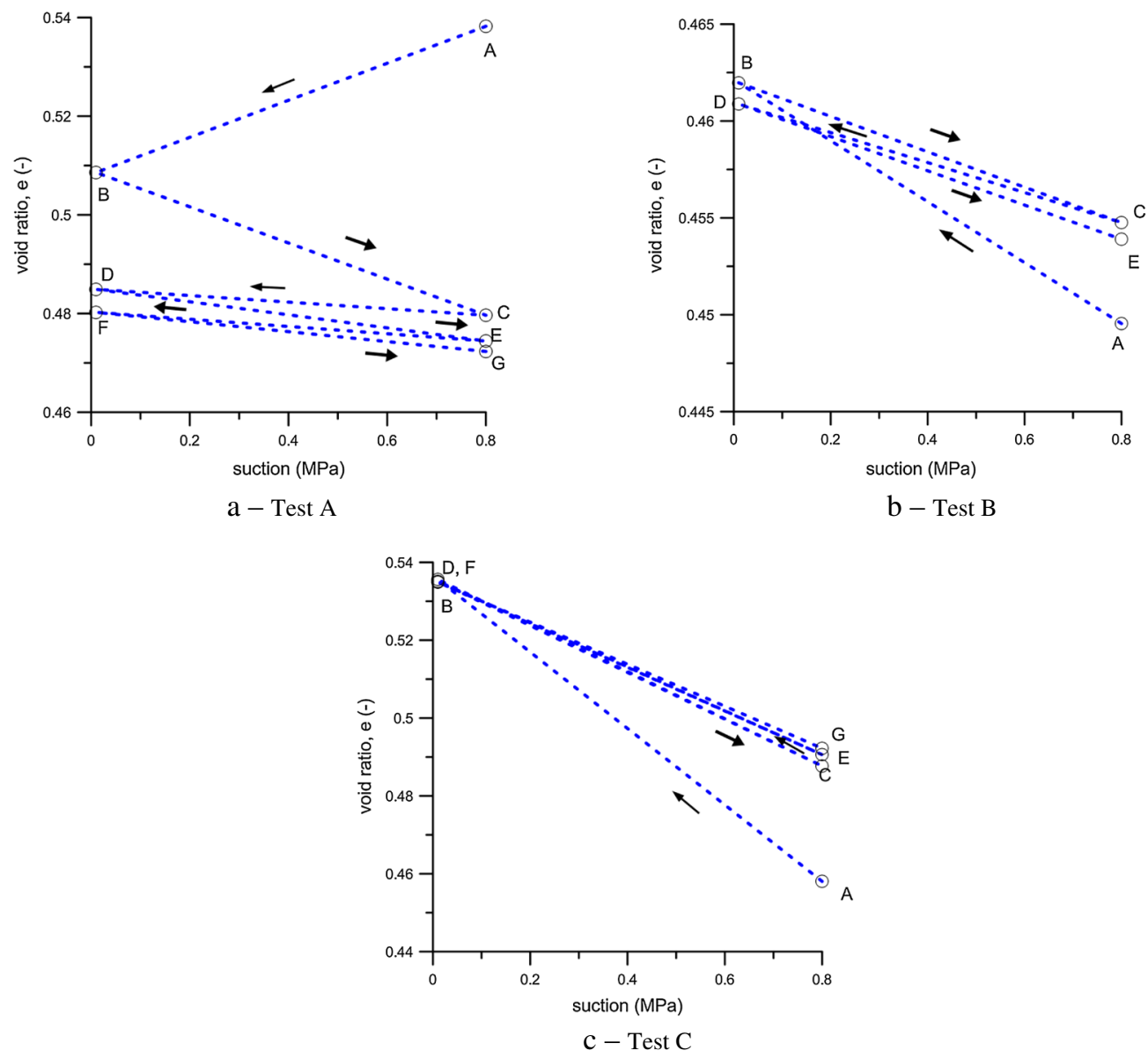

Figure 14. Suction-void ratio relation for Sicilian scaly clay [55].

Compressive strains accumulate as a consequence of wetting-drying cycles, most of them occurring along the first wetting-drying cycle. With reference to the hydraulic behaviour, predominant water inflow was observed in the first cycles, which motivated the choice of a hysteretic retention model. Here, the data reported in Figure 14 have been used as an input of the calibrated water retention model, whose predictions in terms of water ratio variation are shown in Figure 15. A linear relation between degree of saturation and suction was assumed for the scanning region, as discussed by Della Vecchia et al. [33].

Further evidence of the different hydraulic responses of the material depending on suction value (or water ratio) is presented in Figure 16, where experimental data from Airò Farulla et al. [54] and numerical prediction of a free-swelling test at decreasing suction are compared. For each suction and void ratio value, the estimated water ratio along main wetting path has been calculated: changes in the swelling rate with suction are predicted very well by the model. 


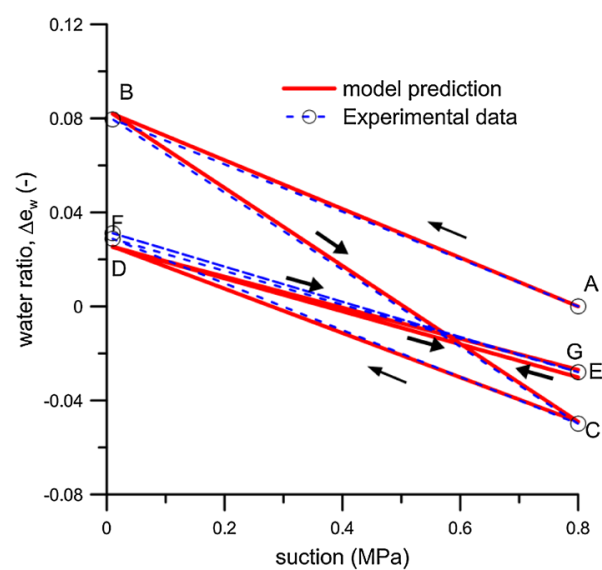

$\mathrm{a}-$ Test A

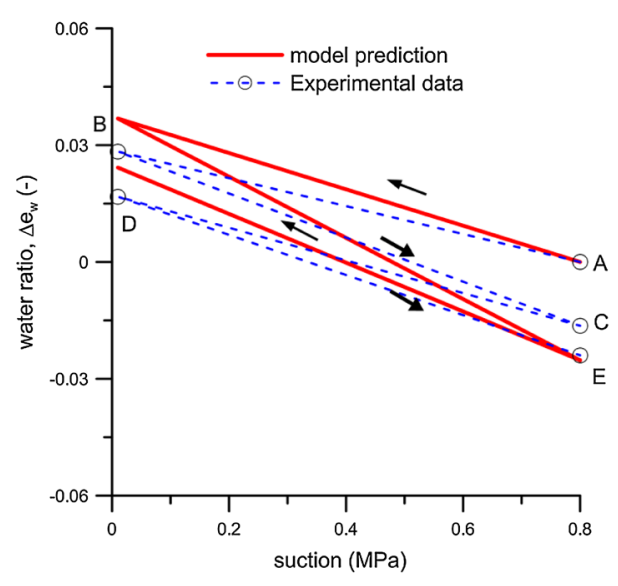

$\mathrm{b}-$ Test B

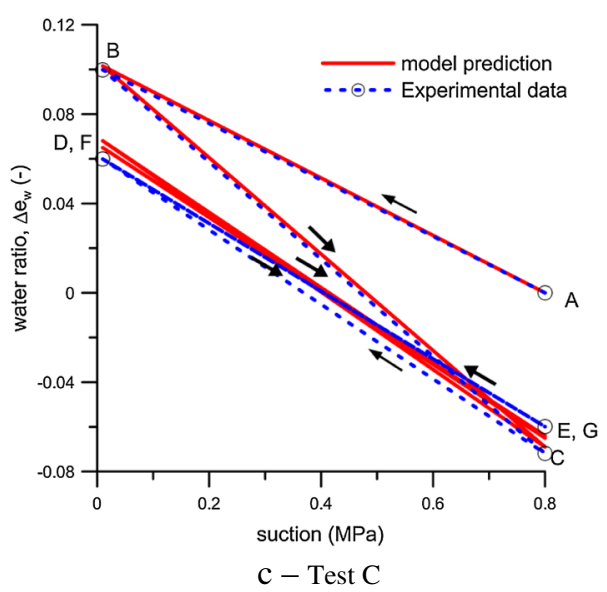

Figure 15. Suction-water ratio relation for Sicilian scaly clay [55]: experimental data and model predictions.

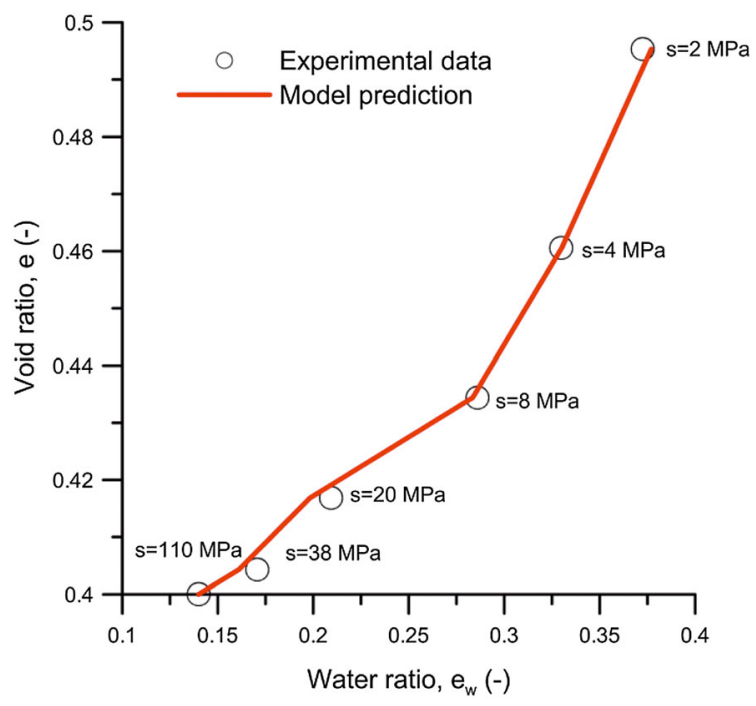

Figure 16. Free-swelling test: experimental data versus model prediction. Experimental data from Airò Farulla et al. [54]. 


\section{CONCLUSIONS}

A framework for modelling the retention behaviour of compacted clayey soils, able to take into account the PSD function evolution along hydraulic and mechanical paths, is discussed. Starting from the theoretical link between the distribution of pore radii in a porous medium and its water retention curve, a methodology was introduced which proves able to translate the quantitative information retrieved from MIP into evolution laws for water retention properties. The framework, based on experimental PSD data, allows discussing several features related to the influence of micro-fabric evolution along hydro-mechanical paths, with the following conclusions: (i) aggregate porosity depends on water content evolution; (ii) the air-entry level of micro-pores evolves as a function of microstructural void ratio; (iii) the air-entry level of macro-pores is linked to both microstructural and global void ratio. Remarkably, the evolution law for the air-entry level of intra-aggregate pores can be approximately considered unique, at least for clays from low to medium activity. As a further tool to increase the applicability of the framework to engineering problems, that is, to reduce the number of parameters to calibrate, correlations between the two parameters ruling the aggregate size evolution and the activity of the clayey soil have been proposed.

The framework has been implemented in practice by using two curves of the van Genuchten type to describe the water retention properties of the two different porosity levels. The introduction of the evolution laws for both aggregate size and PSDs allows the water retention model to follow step by step the evolving structure of the material. As a crucial consequence, the model proved able to follow the transition between bimodal and unimodal PSDs along wetting paths, highlighting the nature of the water retention curve as the subset of hydraulic states belonging to a material with a different microstructure for each water content value. The capability of including microstructure evolution as a consequence of both water content and void ratio variation was exploited also to predict the different water retention responses of a porous medium subjected to various mechanical constraints.

The model was finally validated on the basis of experimental data for a compacted Sicilian scaly clay. Calibration of the parameters took advantage of the correlations previously derived and was performed based on one main drying and one main wetting retention curves at a constant void ratio, without using any further information from specific MIP.

Several aspects of the hydraulic response of the compacted clayey soils were coherently modelled, namely the influence of void ratio on main drying and main wetting curves, the water exchanged along cyclic suction changes and the suction-dependent swelling rate upon hydration. The proposed physically based conceptual framework can thus be used as a tool for predicting the hydraulic response of compacted clay along general hydro-mechanical path, including most situations that can be found in engineering applications.

Simple extensions to include other aspects that can influence the water retention properties of a clayey soil, such as the presence of a shielding skeleton [24, 38, 53], the chemical composition of the pore fluid [17] and the effects of micro-biological activity [58], could be provided, thanks to the generality of the conceptual framework and the physical base of the state variables introduced.

\section{APPENDIX A.}

Without loss in conceptual generality, the framework proposed is here applied assuming that the hydraulic response of each structural level can be modelled by a water retention curve of the van Genuchten type:

$$
G(\psi)=\operatorname{Sr}(\psi)=\left[\frac{1}{1+(\alpha \psi)^{n}}\right]^{m}
$$

Given the general definitions in Section 2, the PSD of each structural level of the porous medium can be derived as follows: 


$$
\begin{aligned}
F^{e}(R) & =e S r(R) \\
f^{e}(r) & =\frac{d F^{e}(r)}{d r} \\
P S D & =\frac{d F^{e}(r)}{d \log (r)}=f^{e}(r) \cdot r \cdot \ln (10)
\end{aligned}
$$

being $F^{e}(R)$ the portion of void ratio corresponding to pores whose size is smaller than $R$.

From Equation (A.1), the previously defined functions can be expressed analytically as

$$
\begin{aligned}
F^{e}(R) & =e\left[\frac{1}{1+\left(\frac{\alpha C}{R}\right)^{n}}\right]^{m} \\
f^{e}(r) & =e \cdot n \cdot m \cdot \alpha \cdot C \cdot \operatorname{Sr}(r) \cdot \frac{\left(\frac{\alpha C}{r}\right)^{n-1}}{\left[1+\left(\frac{\alpha C}{r}\right)^{n}\right]} \cdot \frac{1}{r^{2}} \\
\operatorname{PSD}(r) & =e \cdot n \cdot m \cdot \alpha \cdot C \cdot \operatorname{Sr}(r) \cdot \frac{\left(\frac{\alpha C}{r}\right)^{n-1}}{\left[1+\left(\frac{\alpha C}{r}\right)^{n}\right]} \cdot \frac{1}{r} \cdot \ln (10)
\end{aligned}
$$

If a double-porosity medium is considered, characterised by micro (m)-pores and macro (M)-pores, the following relations hold for $F^{e}(R), f^{e}(r)$ and $\operatorname{PSD}(r)$ :

$$
\begin{aligned}
F^{e}(R) & =e S r(R)=e_{m} S r^{m}(R)+e_{M} S r^{M}(R) \\
f^{e}(r) & =\frac{d F^{e}(r)}{d r}=e_{m} \frac{d S r^{m}(r)}{d r}+e_{m} \frac{d S r^{M}(r)}{d r} \\
P S D & =\frac{d F^{e}(r)}{d \log (r)}=f^{e}(r) \cdot r \cdot \ln (10)
\end{aligned}
$$

Assuming that the link between $S r$ and $\psi$ is given by the van Genuchten equation for each structural level (Equation (14)), the previously defined function can be expressed analytically as

$$
\begin{aligned}
F^{e}(R) & =e_{m}\left[\frac{1}{1+\left(\alpha_{m} \frac{C}{R}\right)^{n_{m}}}\right]^{m_{m}}+e_{M}\left[\frac{1}{1+\left(\alpha_{M} \frac{C}{R}\right)^{n_{M}}}\right]^{m_{M}} \\
f^{e}(r) & =f^{e m}(r)+f^{e M}(r)=e_{m} \frac{d S r^{m}(r)}{d r}+e_{M} \frac{d S r^{M}(r)}{d r} \\
P S D & =\frac{d F^{e}(r)}{d \log (r)}=f^{e m}(r) \cdot r \cdot \ln (10)+f^{e M}(r) r \cdot \ln (10)
\end{aligned}
$$

The corresponding water retention model is finally expressed in terms of water ratio as

$$
e_{w}(\psi)=e_{w m}+e_{w M}=e_{m}\left[\frac{1}{1+\left(\alpha_{m} \psi\right)^{n_{m}}}\right]^{m_{m}}+\left(e-e_{m}\right)\left[\frac{1}{1+\left(\alpha_{M} \psi\right)^{n_{M}}}\right]^{m_{M}}
$$




\section{ACKNOWLEDGEMENTS}

The first author wishes to acknowledge Andrea Casafina for his help in performing some of the simulations presented. The financial support of the Italian Ministry of Education and Scientific Research through grant PRIN 2010SWTCKC_002 is also acknowledged by G. Della Vecchia and C. Jommi.

\section{REFERENCES}

1. Brooks RH, Corey AT. Hydraulic properties of porous media. Hydrological Papers (Colorado State University) 1964; 3.

2. van Genuchten MT. A closed-form equation for predicting the hydraulic conductivity of unsaturated soils. Soil Science Society of America Journal 1980; 44:892-898.

3. Fredlund DG, Xing A. Equations for the soil-water characteristic curve. Canadian Geotechnical Journal 1994; 31 (3):521-532.

4. Huang S, Barbour SL, Fredlund DG. Development and verification of a coefficient of permeability function for a deformable unsaturated soil. Canadian Geotechnical Journal 1998; 35(3):411-424.

5. Vanapalli SK, Fredlund DG, Pufahl DE. The influence of soil structure and stress history on the soil-water characteristics of a compacted till. Geotechnique 1999; 49(2):143-159.

6. $\mathrm{Ng} \mathrm{CW}$, Pang YW. Influence of stress state on soil-water characteristics and slope stability. Journal of Geotechnical and Geoenvironmental Engineering 2000; 126(2):157-166.

7. Kawai K, Kato S, Karube D. The model of water retention curve considering effects of void ratio. Proceedings of the Asian Conference on Unsaturated Soils, UNSAT-ASIA 2000, Singapore, 18-19 May, 2000. In Unsaturated Soils for Asia. Rahardjo H, Toll DG, Leong EG (eds.). AA. Balkema, Rotterdam, 2000; 329-334.

8. Gallipoli D, Wheeler SJ, Karstunen M. Modelling the variation of degree of saturation in a deformable unsaturated soil. Geotechnique 2003; 53(1):105-112.

9. Sun DA, Sheng D, Sloan SW. Elastoplastic modelling of hydraulic and stress-strain behaviour of unsaturated soils. Mechanics of Materials 2007; 39:212-221.

10. Nuth M, Laloui L. Advances in modelling hysteretic water retention curve in deformable soils. Computers and Geotechnics 2008; 35:835-844.

11. Tarantino A. A water retention model for deformable soils. Geotechnique 2009; 59(9):751-762.

12. Mašín D. Predicting the dependency of a degree of saturation on void ratio and suction using effective stress principle for unsaturated soils. International Journal for Numerical and Analytical Methods in Geomechanics 2010; 34(1):73-90.

13. Sheng D, Zhou AN. Coupling hydraulic with mechanical models for unsaturated soils. Canadian Geotechnical Journal 2011; 48:826-840.

14. Gallipoli D. A hysteretic soil-water retention model accounting for cyclic variations of suction and void ratio. Geotechnique 2012; 62(7):605-616.

15. Zhou AN, Sheng D, Carter JP. Modelling the effect of initial density on soil-water characteristic curves. Geotechnique 2012; 62(8):669-680.

16. Thom R, Sivakumar R, Sivakumar V, Murray E, MacKinnon PA. Pore size distribution of unsaturated compacted kaolin: the initial states and final states following saturation. Geotechnique 2007; 57(7):469-474.

17. Musso G, Romero E, Della Vecchia G. Double-structure effects on the chemo-hydro-mechanical behaviour of a compacted active clay. Geotechnique 2013; 63(3):206-220.

18. Delage P. A microstructure approach to the sensitivity and compressibility of some Eastern Canada sensitive clays. Geotechnique 2010; 60(5):353-368.

19. Romero E, Gens A, Lloret A. Water permeability, water retention and microstructure of unsaturated Boom clay. Engineering Geology 1999; 54:117-127.

20. Cuisinier O, Laloui L. Fabric evolution during hydromechanical loading of a compacted silt. International Journal for Numerical and Analytical Methods in Geomechanics 2004; 28(6):483-499.

21. Koliji A, Laloui L, Cuisinier O, Vulliet L. Suction induced effects on the fabric of a structured soil. Transport in Porous Media 2006; 64:261-278.

22. Della Vecchia G. Coupled hydro-mechanical behaviour of compacted clayey soils. Phd Thesis, Politecnico di Milano, 2009.

23. Monroy R, Zdravkovic L, Ridley A. Evolution of microstructure in compacted London Clay during wetting and loading. Geotechnique 2010; 60(2):105-119. doi:10.1680/geot.8.P.125.

24. Wang Q, Tang AM, Cui YJ, Delage P, Gatmiri B. Experimental study on the swelling behaviour of bentonite/claystone mixture. Engineering Geology 2012; 124(4):59-66.

25. Delage P, Lefebvre G. Study of the structure of a sensitive Champlain clay and its evolution during consolidation. Canadian Geotechnical Journal 1984; 21:21-35.

26. Griffiths FJ, Joshi RC. Change in pore size distribution due to consolidation of clays. Geotechnique $1989 ; 39$ (1):159-167.

27. Al-Mukhtar M, Belanteur N, Tessier D, Vanapalli SK. The fabric of a clay soil under controlled mechanical and hydraulic stress states. Applied Clay Science 1996; 11(2):99-115.

28. Simms PH, Yanful EK. Estimation of soil-water characteristic curve of clayey till using measured pore-size distribution. Journal of Environmental Engineering 2004; 130(8):847-854. 
29. Simms PH, Yanful EK. Predicting soil-water characteristic curves of compacted plastic soils from measured poresize distributions. Geotechnique 2002; 52(4):269-278.

30. Romero E, Della Vecchia G, Jommi C. An insight into the water retention properties of compacted clayey soils. Geotechnique 2011; 61(4):313-328.

31. Casini F, Vaunat J, Romero E, Desideri A. Consequences on water retention properties of double-porosity features in a compacted silt. Acta Geotechnica 2012; 7(2):139-150.

32. Beckett CT, Augarde CE. Prediction of soil water retention properties using pore-size distribution and porosity. $\mathrm{Ca}$ nadian Geotechnical Journal 2013; 50(4):435-450.

33. Della Vecchia G, Jommi C, Romero E. A fully coupled elastic-plastic hydromechanical model for compacted soils accounting for clay activity. International Journal for Numerical and Analytical Methods in Geomechanics 2013; 37 (5):503-535.

34. Hu R, Chen YF, Liu HH, Zhou CB. A water retention curve and unsaturated hydraulic conductivity model for deformable soils: consideration of the change in pore-size distribution. Geotechnique 2013; 63(16):1389-1405.

35. Dieudonné AC, Levasseur S, Charlier R, Della Vecchia G, Jommi C. A water retention model for compacted clayey soils. In Computational Geomechanics COMGEO III, Proceedings of the 3rd International Symposium on Computational Geomechanics, Krakow, Poland, Pietruszczak S, Pande GN (eds.). International Centre for Computational Engineering: Rhodes, Greece \& Swansea, UK, 2013; 23-31.

36. Millington RJ, Quirk JP. Permeability of porous media. Nature 1961; 1983:387-388.

37. Mualem Y. A new model for predicting the hydraulic conductivity of unsaturated porous media. Water Resources Research 1976; 12(3):513-522.

38. Wang Q, Tang AM, Cui YJ, Delage P, Barnichon JD, Ye WM. The effects of technological voids on the hydromechanical behaviour of compacted bentonite-sand mixture. Soils and Foundations 2013; 53(2):232-245.

39. Saba S, Delage P, Lenoir N, Cui YJ, Tang AM, Barnichon JD. Further insight into the microstructure of compacted bentonite-sand mixture. Engineering Geology 2014; 168:141-148.

40. Lloret A, Villar MV, Sánchez M, Gens A, Pintado X, Alonso EE. Mechanical behaviour of heavily compacted bentonite under high suction changes. Geotechnique 2003; 53(1):27-40.

41. Tarantino A, De Col E. Compaction behaviour of clay. Geotechnique 2008; 58(3):199-213.

42. Buenfil C, Romero E, Lloret A, Gens A. Hydro-mechanical behaviour of a clayey silt under isotropic compression. Springer Proceedings in Physics 2005; 93(4):331-342.

43. Romero E, Simms P. Microstructure investigation in unsaturated soils: a review with special attention to contribution of mercury intrusion porosimetry and environmental scanning electron microscopy. Geotechnical and Geological Engineering 2008; 26:705-722.

44. Lloret A, Villar MV. Advances on the knowledge of the thermo-hydro-mechanical behaviour of heavily compacted "FEBEX" bentonite. Physics and Chemistry of the Earth, Parts A/B/C 2007; 32(8):701-715.

45. Cui YJ, Loiseau C, Delage P. Microstructure changes of a confined swelling soil due to suction controlled hydration. In Proc. ${ }^{\text {rd }}$ Int. Conf. on Unsaturated Soils, Recife, Brazil, 10-13 March 2002. Jucá JFT, de Campos TMP, Marinho FAM (eds.). A.A. Balkema Publishers, Lisse, vol. 2, 2002; 593-598.

46. Prapaharan S, Altschaeffl AG, Dempsey BJ. Moisture curve of a compacted clay: mercury intrusion method. Journal of Geotechnical Engineering, ASCE 1985; 111(9):1139-1143.

47. Simms PH, Yanful EK. Measurement and estimation of pore shrinkage and pore distribution in a clayey till during soil-water characteristic curve tests. Canadian Geotechnical Journal 2001; 38:741-754.

48. Delage P, Marcial D, Cui YJ, Ruiz X. Ageing effects in a compacted bentonite: a microstructure approach. Geotechnique 2006; 56(5):291-304.

49. Zhang LM, Li X. Microporosity structure of coarse granular soils. Journal of Geotechnical and Geoenvironmental Engineering 2010; 136(10):1425-1436.

50. Mašín D. Double structure hydromechanical coupling formalism and a model for unsaturated expansive clays. Engineering Geology 2013; 165:73-88.

51. Loiseau C, Cui YJ, Delage P. The gradient effect on the water flow through a compacted swelling soil. In Proceedings of the 3rd International Conference on Unsaturated Soils (UNSAT 2002), Recife, Brazil, Jucá JFT, de Campos TMP, Marinho FAM (eds.). vol. 1, 2002; Swets \& Zeitlinger, Lisse.

52. Cui YJ, Tang AM, Loiseau C, Delage P. Determining the unsaturated hydraulic conductivity of a compacted sand-bentonite mixture under constant-volume and free-swell conditions. Physics and Chemistry of the Earth 2008; 33:S462-S471.

53. Romero E. A microstructural insight into compacted clayey soils and their hydraulic properties. Engineering Geology 2013; 165:3-19.

54. Airò Farulla C, Battiato A, Ferrari A. The void ratio dependency of the retention behaviour for a compacted clay. Proc. $5^{\text {th }}$ Int. Conf. on Unsaturated Soils, Barcelona, Spain. In Unsaturated Soils, Alonso \& Gens (ed.). Taylor \& Francis Group: London, 2011; 417-422.

55. Airò Farulla C. Stress path dependence of hydromechanical behaviour of compacted scaly clay in wetting and drying suction controlled oedometer tests at constant vertical net stress. Proc. $1^{\text {st }}$ Eur. Conf. on Unsaturated Soils, Durham, UK. In Unsaturated Soils: Advances in Geo-engineering, Toll DG et al. (eds). Taylor \& Francis Group: London, 2008; 321-326.

56. Gens A, Alonso EE. A framework for the behaviour of unsaturated expansive clays. Canadian Geotechnical Journal 1992; 29:1013-1032. 
57. Alonso E, Vaunat J, Gens A. Modelling the mechanical behaviour of expansive clay. Engineering Geology 1999; $\mathbf{5 4 : 1 7 3 - 1 8 3 . ~}$

58. Morales L, Romero E, Jommi C, Garzón E, Giménez A. Feasibility of a soft biological improvement of natural soils used in compacted linear earth construction. Acta Geotechnica 2014; 1-15, on line, doi:10.1007/s11440-014-0344-x.

59. Wang Q. Hydro-mechanical behaviour of bentonite-based materials used for high-level radioactive waste disposal. Phd Thesis. Ecole des Pont, Paristech. Paris, France, 2012. 